\title{
Dermatofibrosarcoma protuberans with pit-like lesions: A case report and literature review
}

\author{
JINJING JIA，YAN ZHENG，XINYU DONG，XIN WANG，JIAO YANG，ZHAOXIA YING， \\ YONGXIAN WANG, XINWU NIU and SHENGXIANG XIAO
}

\begin{abstract}
Department of Dermatology, Second Affiliated Hospital of Xi'an Jiaotong University, Xi'an, Shaanxi 710004, P.R. China
\end{abstract}
Received October 10, 2014; Accepted July 7, 2015

DOI: $10.3892 / 01.2015 .3741$

\begin{abstract}
The present study describes a case of pit-like dermatofibrosarcoma protuberans (DFSP) with the clinical manifestations, histopathological features and criteria for diagnosis. The study also reviews the relevant literature in order to raise awareness among dermatologists with regard to the specific behavior of DFSP. A 27-year-old female presented with subcutaneous nodules on the left side of the neck that had been apparent for 5 years and which had gradually begun caving in during the last year. Histopathological examination revealed that the tumor was composed of a large number of spindle cells arranged in a whirlpool-like pattern. Immunohistochemical studies revealed positive staining for cluster of differentiation 34, vimentin and lysozyme, which could be used as diagnostic markers of DFSP. The patient was finally diagnosed with DFSP by pathological and immunohistochemical analyses. The DFSP was treated with an extended resection followed by adjacent skin repair. The patient responded well and no relapse occurred during the 8-month clinical follow-up. Thus, the current study describes a unique pit-like clinical manifestation of DFSP with typical immunohistochemical and pathological features. In addition, histopathological examination revealed a downward depression in the epidermis. Therefore, histopathological examination should be considered as an essential diagnostic tool for DFSP.
\end{abstract}

\section{Introduction}

Dermatofibrosarcoma protuberans (DFSP) is a localized, low-grade malignant fibrosarcoma originating in the skin and extending into the subcutaneous tissue (1). It is a rare tumor, accounting for only $0.1 \%$ of all cutaneous malignancies, with an overall incidence of $0.8-5.0$ cases per million individuals in the

Correspondence to: Dr Yan Zheng, Department of Dermatology, Second Affiliated Hospital of Xi'an Jiaotong University, 157 Xiwu Road, Xi'an, Shaanxi 710004, P.R. China

E-mail: zenyan66@126.com

Key words: eminence skin fibrosarcoma, pit-like, cluster of differentiation 34, vimentin, lysozyme
USA (2). It is associated with a low capacity for infiltration, rare occurrences of metastasis and a high incidence of local recurrence. Clinically, this tumor often presents as a protuberant and hard solid lump. The diagnosis depends on the histopathological and immunohistochemical examination (3). Surgical resection is the first choice for treatment and the prognosis is relatively good, with a five-year survival rate of $>90 \%$ (4). However, the local recurrence rate is high, ranging between 0 and $60 \%$ depending on the surgical technique used and tumor location (5). Therefore, early diagnosis and complete resection are extremely important. To the best our knowledge, no case of pit-like DFSP has previously been reported. The present study reports one such case of pit-like DFSP in a female patient.

\section{Case report}

A 27-year-old female presented to the Second Affiliated Hospital of Xi'an Jiaotong University (Xi'an, Shaanxi, China) with subcutaneous nodules on the left side of the neck, which had been present for 5 years. The nodules had gradually begun caving in during the last year. The patient had incidentally noticed a nodule the size of a soybean and of normal skin color that was not elevated above the skin surface. Since there were no other symptoms, the nodule was not considered to be serious. Thereafter, the patient often habitually touched and pressed the nodule, which slowly increased in size. At $\sim 1$ year prior to presentation, a mild depression was noted on the nodule surface and the area became pink in color. As the depression progressively deepened, the patient visited the hospital. The nodule was surgically resected and submitted for pathological examination. The nodule appearance is shown in Fig. 1. On the left side of the neck, an irregular depression $(2.5 \mathrm{~cm}$ in length, $1.0 \mathrm{~cm}$ in width and $\sim 0.3 \mathrm{~cm}$ in depth) with an unclear boundary was observed (Fig. 1). A subcutaneous nodule with a rubbery consistency was palpable; it was not tender, showed little activity and adhered to the subcutaneous tissue.

Histopathological examination (Fig. 2) revealed visible depression in the skin lesions, with normal tissue on the right side of the neck raised higher than the nodular tissue on the left side. The epidermis appeared normal. Tissue sections from the tumor located between the sunken dermis and subcutaneous tissue showed the presence of a non-capsulated 


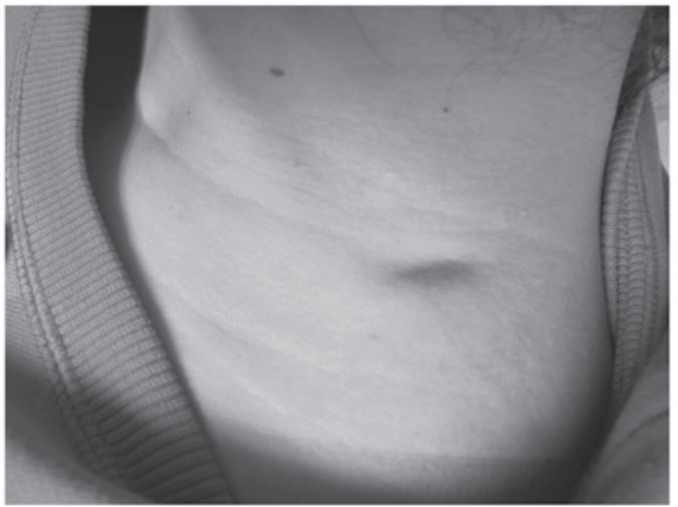

Figure 1. Appearance of the nodule in the patient's neck. An irregular depression $(2.5 \mathrm{~cm}$ in length, $1.0 \mathrm{~cm}$ in width and $\sim 0.3 \mathrm{~cm}$ in depth) with an unclear boundary was present in the left side of the neck.

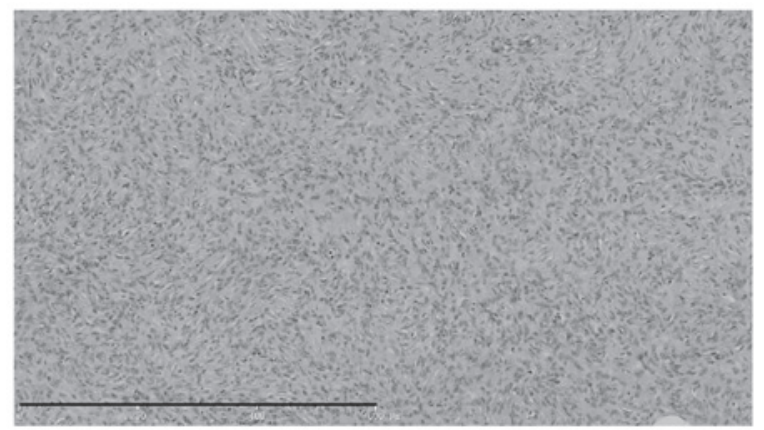

Figure 2. Histopathological examination. Fusiform cells with large nuclei were interwoven into a whirlpool pattern, with little nuclear mitotic activity (hematoxylin-eosin staining; scale bar, $600 \mu \mathrm{m}$ ).

tumor composed of fusiform cells with large nuclei that were interwoven into a whirlpool pattern, with little nuclear mitotic activity, but with fat segmentation.

Immunohistochemical studies revealed positive staining for cluster of differentiation (CD)34, vimentin, and lysozyme (Fig. 3), but negative staining for CD31, CD68, S100, factor VIII, desmin and smooth muscle actin (SMA) (Fig. 4). The diagnosis of DFSP was based on the aforementioned findings. The patient then underwent an extended resection of the tumor, followed by adjacent skin repair. The resected tumor was further examined histopathologically, and no tumor involvement was detected at the surgical margins. Following surgery, the patient achieved good results, with no relapse during the 8-month clinical follow-up period.

\section{Discussion}

DFSP was first described in 1890 as a rare tumor originating from fibroblasts and myofibroblasts with a keloid-like appearance and a tendency for recurrence (6). The etiology of DFSP remains unclear and may be associated with certain genetic characteristics, a history of trauma and the extent of radiation exposure. Recent studies on the molecular basis of DFSP development have reported that $>90 \%$ of DFSP patients present with the $\mathrm{t}(17 ; 22)(\mathrm{q} 22 ; \mathrm{q} 13.1)$ chromosomal translocation, which causes the fusion of the collagen type $1 \alpha 1$ chain
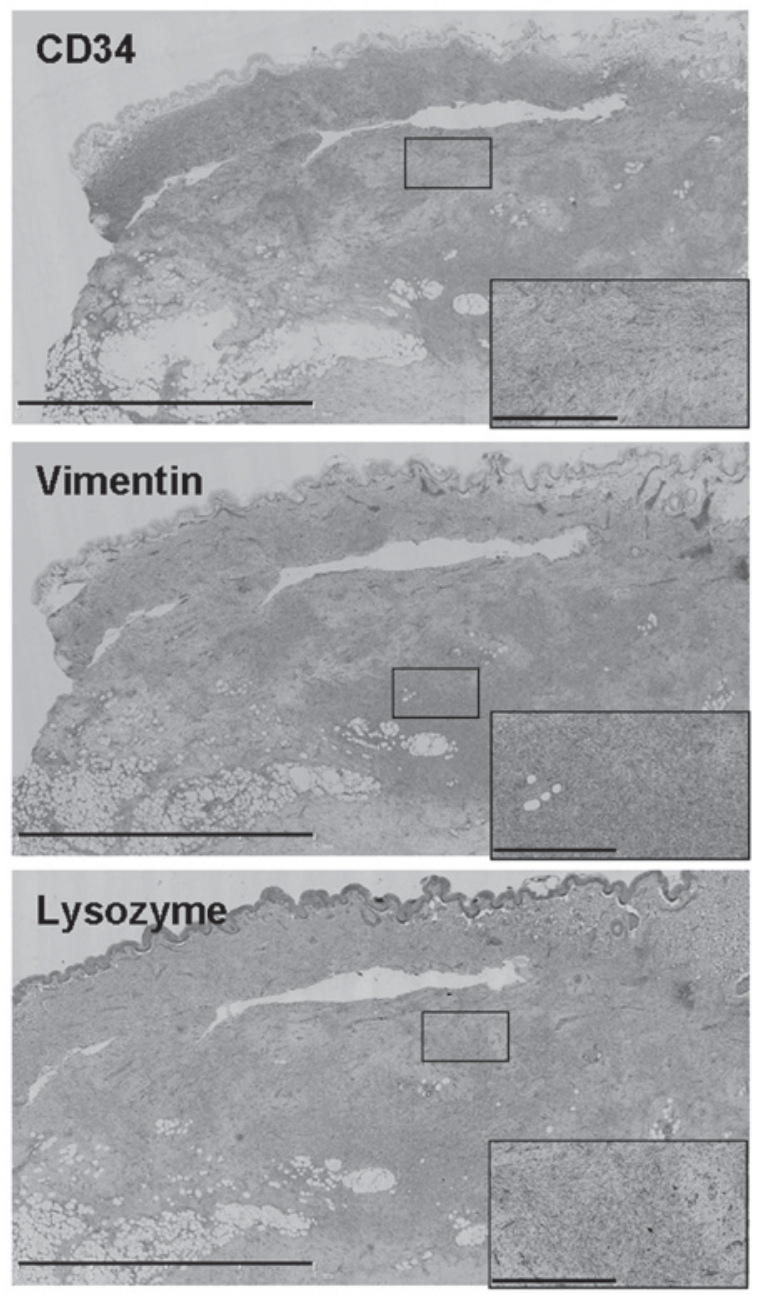

Figure 3. Positive staining for cluster of differentiation 34, vimentin and lysozyme by immunohistochemical assay.

(COL1A1) gene on 17q22 and the platelet-derived growth factor $\beta$ chain (PDGFB) gene on 22q13.1 (7-10). This results in the formation of the COL1A1-PDGFB fusion protein. The sustained activation of fusion protein-related receptors by protein tyrosine kinase causes abnormal cell proliferation, thereby resulting in DFSP (11).

Although DFSP can occur at any age, it is more common in adults between 20 and 50 years of age, regardless of gender (12). The tumor often presents in the torso, particularly the chest, followed by the proximal extremities, but rarely occurs in the head and neck region (13). The clinical manifestations of this slow-growing tumor vary, depending on the developmental stage, presenting as pink- or skin-colored nodules with diameters ranging from a few millimeters to a few centimeters (14). The tumor is usually hard to touch and initially adheres to the epidermis or subcutaneous tissue. The disease further progresses with the occurrence of a single nodule or multiple nodules of different sizes and has a protruding appearance. The growth of these nodules can be suddenly accelerated until their surface ruptures (7). DFSP is usually asymptomatic, except for the presence of mild to moderate pain in certain patients. Recurrence following tumor resection is common, and disseminated nodules can occur around the incision (15). However, tumor metastasis is rare. In the advanced stages, the 

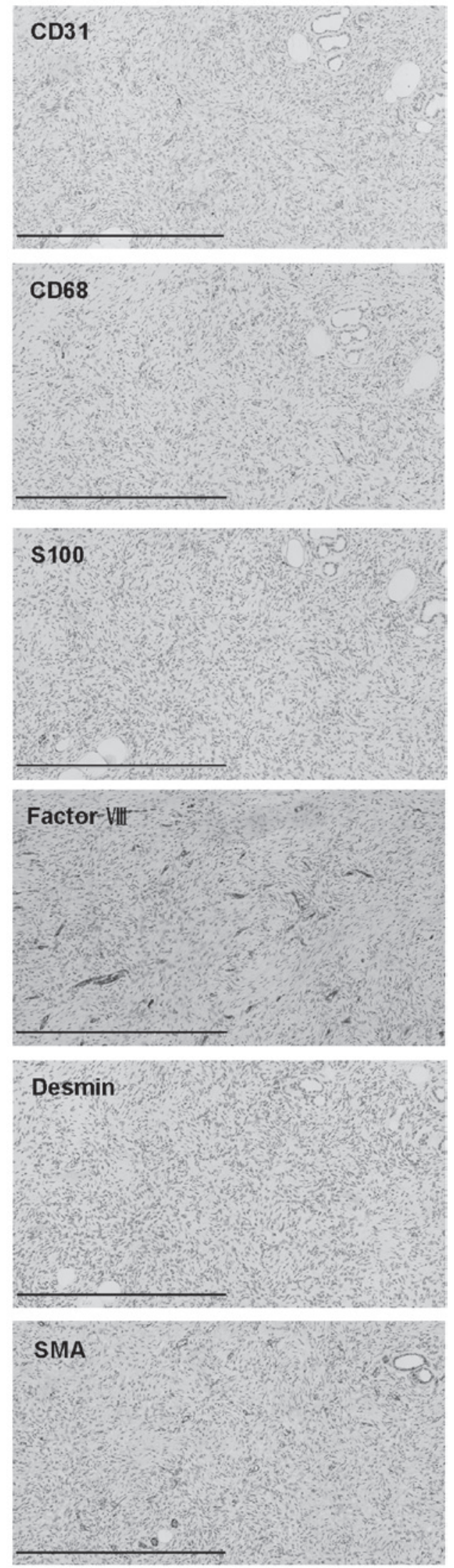

Figure 4. Negative staining for cluster of differentiation (CD)31, CD68, S100, factor VIII, desmin and smooth muscle actin by immunohistochemical assay (scale bar, $600 \mu \mathrm{m}$ ).

disease may occasionally metastasize to the lungs, abdomen, brain, bones or nearby lymph nodes, often due to repeated local recurrence. DFSP can also present as atrophic lesions, sclerotic plaques and localized scleroderma, and hence, it is termed atrophic DFSP (or non-eminent DFSP) $(16,17)$. Such a presentation could easily lead to a misdiagnosis. To the best of our knowledge, a nodule with depressed lesions has not previously been reported.

Typical DFSP are characterized as a tumor without a capsule, located in the dermis and separated from the epidermis by normal narrow bands. Diffuse irregular invasion into the subcutaneous fat, presenting as a lace-like and branch-shaped beams in parallel with epidermal cells, is also typically observed. Tumor histology reveals spindle cells of uniform size with few areas of dual-colored cytoplasm, and stained oval- or spindle-shaped nuclei. The cells appear similar to naive fibroblasts with good differentiation and little mitosis. Furthermore, tumor cells are generally arranged around small blood vessels or fiber axes in a radial, spoke- or whirlpool-like pattern. Such an arrangement is of diagnostic significance. Diffused and positive staining for CD34 with a sensitivity as high as $84-100 \%$ detected by immunohistochemistry is specific for DFSP with diagnostic value (18). Focal positive staining for vimentin, lysozyme, CD68 and SMA has been observed in certain cases, whereas the tumor stains negative for factor VIII, factor XIIIa, S-100 and desmin (18-20). The pathological and immunohistochemical features of the present sturdy were consistent with the aforementioned typical features of DFSF. However, to the best of our knowledge, the pit-like appearance observed in the presence case has not been previously reported.

DFSP is often misdiagnosed as a benign tumor and thus is excised by a routine process, which easily leads to tumor recurrence and even progression to malignant fibrosarcoma or malignant fibrous histiocytoma. Therefore, an early diagnosis of DFSP is extremely important. DFSP diagnosis mostly relies on the histopathological examination. A diagnosis of DFSP should be considered in patients with slowly-growing, painless, single or multiple fused hard nodules in the torso or limbs, and histopathological and immunohistochemical studies should be performed (21). DFSP should also be distinguished from skin fibroma, fibrosarcoma, malignant fibrous histiocytoma and neurofibromatosis (22).

DFSP is generally treated with surgery, particularly with extended resection and Mohs micrographic surgery. Since DFSP easily recurs and presents on the body surface, radiation therapy should not cause serious damage to vital organs and tissues. Certain studies have therefore suggested treating DFSP with a combination of surgery and radiotherapy. However, the tumor should be treated in a timely manner, instead of waiting until repeated recurrences occur, as recurrence can complicate surgery and radiotherapy, and affect the outcome $(23,24)$.

Recent studies on targeted therapy have demonstrated that imatinib mesylate (Gleevec) may be useful in the treatment of DFSP at the molecular level $(21,25,26)$. Imatinib mesylate is a tyrosine kinase inhibitor that can specifically inhibit the expression of PDGF receptor $\beta$, as well as ATP-binding cassette and tyrosine protein kinase (27).

DFSP is a low-grade malignancy with a slow growth rate and good prognosis. The 5- and 15-year survival rates of DFSP can be as high as 99.2 and $97.2 \%$, respectively (28). 
However, its recurrence rate is high and this usually occurs after 3 years of treatment.

In the present study, pathological and immunohistochemical analyses confirmed that the patient had classic DFSP; The tumor was composed of spindles cells exhibiting a whirlpool-like pattern without epidermal atrophy. However, in the present case, the tumor manifested with a pit-like appearance, instead of the commonly observed protuberant lump. Since skin atrophy was not observed, a diagnosis of atrophic DFSP was eliminated. To the best of our knowledge, DFSP with such manifestations has never been reported. Whether the current case presents an early manifestation of DFSP or a novel type of DFSP warrants confirmation by future studies.

\section{Acknowledgements}

The current study was supported by Program for Changjiang Scholars and Innovative Research Team in University (grant no. 1171).

\section{References}

1. Hegde U, Shetty SK, Sreeshyla HS and Doddawada VG: Dermatofibrosarcoma protuberans - a recurrent lesion with unusual presentation in the parotid region. J Clin Diag Res 8: $130-131,2014$

2. Zheng Z, Piao J, Lee JH, Kim SE, Kim SC, Chung KY and Roh MR: Dermatofibrosarcoma protuberans: A study of clinical, pathologic, genetic, and therapeutic features in Korean patients. Yonsei Med J 56: 440-446, 2015.

3. Saiag P, Grob JJ, Lebbe C, et al: Diagnosis and treatment of dermatofibrosarcoma protuberans. European consensus-based interdisciplinary guideline. Eur J Cancer: Jul 16, 2015 (Epub ahead of print).

4. Erdem O, Wyatt AJ, Lin E, Wang $X$ and Prieto VG: Dermatofibrosarcoma protuberans treated with wide local excision and followed at a cancer hospital: Prognostic significance of clinicopathologic variables. Am J Dermatopathol 34: 24-34, 2012

5. Kim M, Huh $\mathrm{CH}$, Cho KH and Cho S: A study on the prognostic value of clinical and surgical features of dermatofibrosarcoma protuberans in Korean patients. J Eur Acad Dermatol Venereol 26: 964-971, 2012.

6. Suit H, Spiro I, Mankin HJ, Efird J and Rosenberg AE: Radiation in management of patients with dermatofibrosarcoma protuberans. J Clin Oncol 14: 2365-2369, 1996.

7. Llombart B, Serra-Guillén C, Monteagudo C, López Guerrero JA, and Sanmartín O: Dermatofibrosarcoma protuberans: A comprehensive review and update on diagnosis and management. Semin Diagn Pathol 30: 13-28, 2013.

8. McArthur G: Molecularly targeted treatment for dermatofibrosarcoma protuberans. Semin Oncol 31: 30-36, 2004.

9. Sjöblom T, Shimizu A, O'Brien KP, Pietras K, Dal Cin P, Buchdunger E, Dumanski JP, Ostman A and Heldin CH: Growth inhibition of dermatofibrosarcoma protuberans tumors by the platelet-derived growth factor receptor antagonist STI571 through induction of apoptosis. Cancer Res 61: 5778-5783, 2001.

10. Kiuru-Kuhlefelt S, El-Rifai W, Fanburg-Smith J, Kere J, Miettinen $\mathrm{M}$ and Knuutila S: Concomitant DNA copy number amplification at $17 \mathrm{q}$ and $22 \mathrm{q}$ in dermatofibrosarcoma protuberans. Cytogenet Cell Genet 92: 192-195, 2001.
11. Qiao J, Patel KU, López-Terrada D and Fang H: Atrophic dermatofibrosarcoma protuberans: Report of a case demonstrated by detecting COL1A1-PDGFB rearrangement. Diagn Pathol 7: 166 , 2012.

12. Loss L and Zeitouni NC: Management of scalp dermatofibrosarcoma protuberans. Dermatol Surg 31: 1428-1433, 2005.

13. Garcia C, Clark RE and Buchanan M: Dermatofibrosarcoma protuberans. Int J Dermatol 35: 867-871, 1996.

14. Llombart B, Monteagudo C, Sanmartin O, López-Guerrero JA, Serra-Guillén C, Poveda A, Jorda E, Fernandez-Serra A, Pellín A, Guillén C and Llombart-Bosch A: Dermatofibrosarcoma protuberans: A clinicopathological, immunohistochemical, genetic (COL1A1-PDGFB) and therapeutic study of low-grade versus high-grade (fibrosarcomatous) tumors. J Am Acad Dermatol 65: 564-575, 2011.

15. Maki RG, Awan RA, Dixon RH, Jhanwar S and Antonescu CR: Differential sensitivity to imatinib of 2 patients with metastatic sarcoma arising from dermatofibrosarcoma protuberans. Int J Cancer 100: 623-626, 2002.

16. HanabusaM,KamoR,Harada Tand Ishii M:Dermatofibrosarcoma protuberans with atrophic appearance at early stage of the tumor. J Dermatol 34: 336-339, 2007.

17. Martin L, Piette F, Blanc P, Mortier L, Avril MF, Delaunay MM, Dréno B, Granel F, Mantoux F, Aubin F, et al: Clinical variants of the preprotuberant stage of dermatofibrosarcoma protuberans. Br J Dermatol 153: 932-936, 2005.

18. Haycox CL, Odland PB, Olbricht SM and Piepkorn M: Immunohistochemical characterization of dermatofibrosarcoma protuberans with practical applications for diagnosis and treatment. J Am Acad Dermatol 37: 438-444, 1997.

19. Dominguez-Malagón HR, Ordóñez NG and Mackay B: Dermatofibrosarcoma protuberans: Ultrastructural and immunocytochemical observations. Ultrastruct Pathol 19: 281-289, 1995.

20. Sachdev R and Sundram U: Expression of CD163 in dermatofibroma, cellular fibrous histiocytoma and dermatofibrosarcoma protuberans: Comparison with CD68, CD34 and Factor XIIIa. J Cutan Pathol 33: 353-360, 2006.

21. Bakry O and Attia A: Atrophic dermatofibrosarcoma protuberans. J Dermatol Case Rep 6: 14-17, 2012.

22. Marque M, Bessis D, Pedeutour F, Viseux V, Guillot B and Fraitag-Spinner S: Medallion-like dermal dendrocyte hamartoma: The main diagnostic pitfall is congenital atrophic dermatofibrosarcoma. Br J Dermatol 160: 190-193, 2009.

23. Bowne WB, Antonescu CR, Leung DH, Katz SC, Hawkins WG, Woodruff JM, Brennan MF and Lewis JJ: Dermatofibrosarcoma protuberans: A clinicopathologic analysis of patients treated and followed at a single institution. Cancer 88: 2711-2720, 2000.

24. Wu JK, Malik MM and Egan CA: Atrophic dermatofibrosarcoma protuberans: An uncommon and misleading variant. Australas J Dermatol 45: 175-177, 2004.

25. Labropoulos SV, Fletcher JA, Oliveira AM, Papadopoulos S and Razis ED: Sustained complete remission of metastatic dermatofibrosarcoma protuberans with imatinib mesylate. Anticancer Drugs 16: 461-466, 2005.

26. Rutkowski P, Debiec-Rychter M, Nowecki Z, Michej W, Symonides M, Ptaszynski K and Ruka W: Treatment of advanced dermatofibrosarcoma protuberans with imatinib mesylate with or without surgical resection. J Eur Acad Dermatol Venereol 25: 264-270, 2011.

27. Price VE, Fletcher JA, Zielenska M, Cole W, Viero S, Manson DE, Stuart M and Pappo AS: Imatinib mesylate: An attractive alternative in young children with large, surgically challenging dermatofibrosarcoma protuberans. Pediatr Blood Cancer 44: 511-515, 2005.

28. Kohli N and Srivastava D: Dermatofibrosarcoma protuberans. In: Dermatology Atlas for Skin of Color. Jackson-Richards D and Pandya AG. Springer, Berlin, pp269-272, 2014. 